\title{
MODELAGENS MIN-MAX-MIN PARA O PROBLEMA DE LOCALIZAÇÃO DE ESTAÇÕES DE RÁDIO BASE
}

\author{
José André de M. Brito * \\ Instituto Brasileiro de Geografia e Estatística (IBGE) \\ Rio de Janeiro - RJ \\ Departamento de Ciência da Computação \\ Centro Universitário Plínio Leite (UNIPLI) \\ Niterói - RJ \\ britom@ibge.gov.br \\ Adilson Elias Xavier \\ Progr. de Eng. de Sistemas e Computação / COPPE \\ Universidade Federal do Rio de Janeiro (UFRJ) \\ Rio de Janeiro - RJ \\ adilson@cos.ufrj.br \\ * Corresponding author / autor para quem as correspondências devem ser encaminhadas \\ Recebido em 01/2005; aceito em 09/2005 após 2 revisões \\ Received January 2005; accepted September 2005 after 2 revisions
}

\begin{abstract}
Resumo
Relata-se uma nova proposta de solução para o problema de localização de estações de rádio base. Tal proposta é baseada na resolução de um problema min-max-min, que possui natureza multinível e é não diferenciável. Para contornar estas dificuldades e poder usar métodos de otimização mais robustos e eficientes, como os métodos de Gradiente e de Newton, transformamos esse problema em um problema diferenciável, usando uma função suavizadora de classe $C^{\infty}$. Assim, a solução é obtida resolvendo-se uma seqüência de subproblemas diferenciáveis que gradualmente aproximam-se do problema original. O uso desta técnica, chamada Suavização Hiperbólica, permite contornar várias das dificuldades apresentadas no problema original. Um algoritmo contendo as essencialidades do método é apresentado. Além da modelagem min-max-min inicial, propomos também três modelagens variantes, que abordam outros aspectos do problema de localização de estações de rádio base. Um conjunto de resultados computacionais é apresentado considerando as modelagens propostas neste trabalho.
\end{abstract}

Palavras-chave: suavização; localização; min-max-min.

\begin{abstract}
We report a new proposal to solve the base station location problem. This proposal is related to a nondifferentiable min-max-min problem with multi-level nature. In order to overcome these difficulties and, thus, to be able to use more robust and efficient optimization tools, such as Gradient and Newton methods, we have developed a smoothing strategy using a special smoothing function of class $\mathrm{C}^{\infty}$. Then, the final solution is obtained by solving a sequence of differentiable sub-problems which gradually approaches the original problem. The use of this technique, called Hyperbolic Smoothing, permits to overcome the main difficulties that arise from the original problem. Besides the initial min-max-min modelling, we propose three variant methods through which other issues of the base station location problem are also taken into account. An algorithm containing the essentialities of the method is also presented together a set of computational results.
\end{abstract}

Keywords: smoothing; location; min-max-min. 


\section{Introdução}

Neste trabalho, propomos uma nova metodologia de solução para o problema que é um dos pontos chave no planejamento de uma rede de telefonia celular.

O problema de localização de estações de rádio base (ERB's) consiste em selecionar, dentro de um conjunto de locais candidatos (região em estudo) à instalação de uma ERB, um subconjunto de custo mínimo e sujeito à cobertura da área em estudo, ao atendimento da demanda e a uma exploração eficiente do espectro de freqüências.

Normalmente, para este processo, não há nenhum modelo de programação matemática que determine quais ERB's devem ser instaladas nos locais candidatos. E sendo assim, o planejamento faz uso apenas de modelos de propagação de sinais (Mateus, 1998).

Dependendo, então, da dimensão da região em estudo, ou seja, do número de pontos a serem atendidos (usuários) e do número de ERB's candidatas à instalação nesta região, o número de soluções possíveis, implementáveis pelo planejamento, pode ser razoavelmente grande. Em virtude disto, o processo de determinação de localização das ERB's utilizado pelo planejamento pode fornecer uma solução apenas razoável, comprometendo em alguns casos a qualidade e os custos com equipamentos.

Face a essa dificuldade, várias abordagens têm sido propostas para a resolução deste problema. Tais abordagens foram implementadas utilizando técnicas de programação inteira (Wolsey, 1998), programação não-linear (Bazaraa et al., 1993), metaheurísticas e heurísticas (Resende, 2004) e teoria dos grafos (Bondy, 1982).

Dentre as inúmeras abordagens existentes para o problema de localização de estações de rádio base, podemos destacar os trabalhos de: Mateus \& Mazzini (2003), que implementaram uma modelagem para o problema de planejamento de uma rede de comunicação móvel em três níveis; Stamatelos \& Epheremides (1996), que consideram o ajuste da localização e da potência das ERB's em ambientes internos; Hanif (1996) que apresenta uma modelagem para o problema de localização de ERB's considerando a propagação do sinal de cada ERB numa região $Q \in \mathfrak{R}^{3}$ e Bhaskar (2000), que utilizando metaheurísticas como Simulated Annealing, Busca Tabu e Algoritmos Genéticos propõe soluções para diversos problemas de telefonia celular.

Todavia, devido às dificuldades intrínsecas a esse problema, mesmo com a utilização dessas técnicas e com a possibilidade da aplicação destas abordagens, a determinação de soluções exatas (Wolsey, 1998) pode ser muito difícil, acarretando apenas na obtenção de soluções viáveis. Estas soluções dependem diretamente das características do problema e da própria ferramenta de otimização utilizada.

Propomos, então, neste trabalho, uma nova abordagem para a solução do problema de localização de estações de rádio base. Essa abordagem está baseada num modelo, de certa forma clássico, cujo desenvolvimento produz um problema min-max-min (Polyak, 1988; Pillo et al., 1993 e Xavier, 2005). Esse problema, por possuir três níveis, e por ser não-linear e não-diferenciável (LeMarechal, 1981) e normalmente de grande porte, é extremamente difícil. A sua resolução nesse trabalho será efetuada através da utilização, em conjunto, das técnicas de suavização (Polyak, 1988; Pillo et al., 1993 e Xavier, 2005) e penalização hiperbólica (Xavier, 2001).

A apresentação deste trabalho está dividida em cinco seções: Na seção 2, temos a descrição do problema de localização de estações de rádio base. Na seção 3, temos o desenvolvimento 
do modelo min-max-min utilizado para resolver o problema de localização de ERB's e a apresentação do algoritmo de suavização hiperbólica. Na seção 4 , temos a apresentação de três modelagens variantes propostas para o problema de localização de ERB's. Na seção 5, temos a apresentação dos resultados computacionais e, na seção 6 , temos as conclusões.

\section{Problema de Localização de Estações de Rádio Base}

\subsection{Definição do problema}

O problema de localização de estações de rádio base (ERB's) aparece como um dos pontos chave no planejamento e desenvolvimento de redes de telefonia celular. Na maioria dos casos, a localização das ERB's é efetuada de uma forma ad hoc pela inspeção de mapas de propagação de sinais (Mateus, 1998 e Stüber, 2000) relacionados à área de estudo. Fornecida uma lista de locais dessa área de estudo onde as estações podem ser localizadas, a idéia é utilizar o conhecimento das características de propagação das ondas de rádio nessa área, de forma a maximizar a cobertura e minimizar os custos.

O problema de localização de estações de rádio base (ERB's) consiste em selecionar, dentro de um conjunto de locais candidatos à instalação de uma ERB, um subconjunto de custo mínimo e sujeito à cobertura da área em estudo e ao atendimento da demanda. Quanto menor o número de ERB's instaladas, menor será o custo. Por outro lado, o atendimento da demanda e a qualidade do serviço podem ser prejudicados.

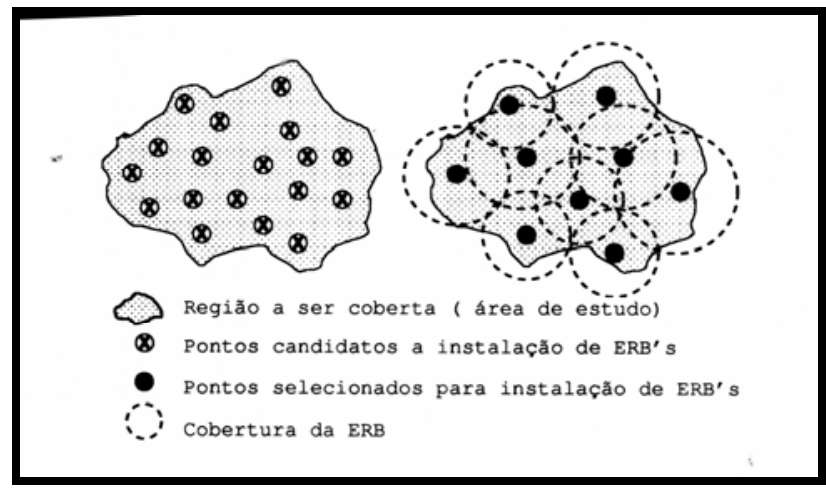

Figura 1 - Exemplo do problema de localização de ERB's.

A localização das estações de rádio base pode estar relacionada a três diferentes objetivos: a cobertura total ou máxima, o máximo aproveitamento espectral e o máximo número de canais por usuário (Mateus, 1998).

A cobertura total consiste em associar a cada ponto da área de estudo pelo menos uma ERB em condições de oferecer um sinal com nível mínimo, que permita a conversação ou a transmissão de dados nesta área.

O máximo aproveitamento espectral busca solucionar as deficiências do problema anterior, limitando a área de cobertura de cada ERB. Nesse caso, a localização das estações de rádio base e suas respectivas potências de transmissão são definidas conjuntamente. 
A determinação dos locais de uma dada região onde haja possibilidade de instalação de ERB's pode ser feita mediante a utilização de um sistema de predição de propagação de sinais (Mateus, 1998).

O método de escolha das ERB's envolve três passos. Inicialmente, as ERB's candidatas são criadas pelo sistema. Parâmetros como altura das antenas, potência de transmissão e localização física da ERB são definidos. O sistema simula, então, a propagação do sinal (Mateus, 1998) de cada ERB ao longo da área de estudo, gerando um mapa de perdas de sinal em cada ponto desta.

No passo seguinte, é feita uma composição dos sinais das diversas ERB's candidatas. Esta composição atribui a cada ponto da área em estudo a ERB de melhor sinal. No terceiro e último passo, as ERB's selecionadas na etapa de composição são submetidas a um algoritmo de alocação de canais, que leva em consideração a demanda de cada ponto da região em estudo e a potência de transmissão de cada ERB. Na fase inicial do projeto, não há uma limitação no número de estações de rádio base que podem ser instaladas na região. $\mathrm{O}$ que existe é uma preocupação com a qualidade do atendimento prestado. A redução desse número de estações é efetuada em uma fase de pós-processamento.

Neste trabalho, trataremos o problema de localização de ERB's levando em conta o aspecto de cobertura total. Dado um número fixo de ERB's, representado por $q$, o objetivo é localizar estas estações de rádio base, ou seja, determinar as suas coordenadas de localização (no plano), de forma a fornecer a cobertura total para a região em estudo utilizando a menor potência de transmissão possível.

Para a resolução desse problema, apresentaremos, na seção três, uma nova modelagem proposta nos trabalhos de Brito \& Xavier (2004) e Xavier (2005) e três modelagens variantes que consideram: a distância máxima entre as estações, considerando que estas estações estão agrupadas, a proximidade das ERB's de pontos pré-determinados e a seleção de locais (pontos) para instalação de ERB's. Observamos que nestas modelagens variantes também é contemplado o aspecto de cobertura total.

Na sessão a seguir, apresentamos, em linhas gerais, algumas das modelagens já existentes para o problema de localização de estações de rádio base.

\subsection{Modelagens clássicas para o problema de localização de ERB’s}

A proposta desta seção é a de fazer uma exposição concisa das abordagens clássicas desenvolvidas para o problema de localização de estações de rádio base, a fim de entender melhor as dificuldades e limitações observadas na solução deste problema. Tais abordagens estão diretamente relacionadas à resolução de modelos que agregam características do problema de set-covering (Padberg, 1979 e Wolsey, 1998).

O set-covering tem a seguinte estrutura geral:

$$
\begin{gathered}
\text { Minimizar } c x \\
A x \geq e^{t} \\
x_{j}=0 \text { ou } x_{j}=1, \quad j=1, \ldots, n
\end{gathered}
$$

onde $A$ é uma matriz ( $\left.\begin{array}{lll}m & x & n\end{array}\right)$ zero-um, $e$ é um vetor com $m$ componentes (de 1's), $c$ é um vetor de custos e $x_{j}$ é uma variável binária (0-1). 
Tal problema é NP-Difícil (Padberg, 1979). Ou seja, dependendo do número de variáveis $x_{j}$, a obtenção de uma solução ótima através de um método de enumeração como Branch and Bound (Wolsey, 1998) pode ser impossível.

Desta forma, várias técnicas têm sido desenvolvidas visando determinar maneiras mais adequadas de enumerar as soluções na busca da solução ótima. Estas técnicas são geralmente de natureza combinatória e se fundamentam na estrutura especial de cada problema analisado. Os algoritmos se concentram em três áreas básicas: decomposição (Geoffrion, 1974 e Rosen, 1983), enumeração (Beasley \& Christofides, 1983 e Mateus et al., 1997) e heurísticas (Resende \& Feo, 1989 e Wolsey \& Fisher, 1982).

A seguir, apresentamos duas modelagens clássicas propostas para a resolução do problema de localização de ERB's. A primeira apresentada em Mateus (1998) e a segunda em Tutschku (1998).

$$
\begin{gathered}
\text { Minimizar } \sum_{i \in N} c_{i} y_{i} \\
\sum_{i \in N} a_{i j} x_{i j} \geq T, \quad \forall j \in M \\
\sum_{i \in N} x_{i j}=1, \quad \forall j \in M \\
x_{i j} \leq y_{i}, \quad \forall i \in N, \forall j \in M \\
y_{i}, x_{i j} \in\{0,1\}, \forall i \in N, \forall j \in M
\end{gathered}
$$

$y_{i}=1 \quad$ se a ERB $i$ é instalada e zero caso contrário.

$x_{i j}=1$ se um ponto $j$ da região é coberto pela ERB $i$ e zero caso contrário.

Para este modelo, os parâmetros de entrada considerados são: o número $M$ de pontos a serem cobertos na região em estudo e o número $N$ de estações de rádio base candidatas para se instalar na região em estudo. Temos também o custo $c_{i}$ de instalação de cada ERB $i, o$ valor mínimo para a medida do sinal capaz de viabilizar a comunicação definido por $T$ e a estimativa do sinal da ERB $i$ no ponto $j$, definida por $a_{i j}$.

A função objetivo minimiza o custo de instalação das ERB's. A restrição (1) garante que pelo menos uma ERB atende à qualidade do sinal no nível mínimo estipulado. A restrição (2) associa a cada ponto da região a ser coberta uma única ERB. A restrição (3) permite esta associação somente se uma ERB for instalada e a (4) representa as restrições de integralidade.

O modelo acima possui um elevado número de variáveis inteiras $(|\mathrm{N}|+|\mathrm{N}| \cdot|\mathrm{M}|)$. Mesmo para pequenas áreas as dimensões do problema tornam-se bastante elevadas, com a geração de milhares de variáveis $x_{i j}$.

Tutschku (1998) apresenta a uma modelagem similar, porém mais compacta, para o problema de localização de ERB's: 


$$
\begin{array}{ll}
\text { Minimizar } & \sum_{j \in J} x_{j} \\
\sum_{j \in N_{i}} x_{j} \geq 1, \quad \forall i \in I
\end{array}
$$

Nessa formulação, $I$ corresponde ao conjunto de pontos da região que deverão ser atendidos pelas ERB's e $N_{i}=\left\{j \mid f_{i j} \leq P L\right\}$ ao conjunto de estações de rádio base $j$ que podem cobrir os pontos $i$ da região, considerando que a perda (atenuação) do sinal da ERB $j$ no ponto $i$ (representada por $f_{i j}$ ) não ultrapasse um certo patamar $P L$.

Convencionando que $x_{j}=1$ corresponde à ERB $j$ ser instalada e $x_{j}=0$ caso contrário, temos que a função objetivo minimiza o número de estações necessárias para cobrir a região e a restrição acima garante que, em uma solução viável, cada ponto da região é atendido por pelo menos uma estação de rádio base.

Este modelo é mais simples, tendo em vista que o número de variáveis inteiras torna-se reduzido, mas como observado inicialmente, está associado a um problema de recobrimento, que é NP-Difícil. No entanto, a matriz formada pelos $f_{i j}$ pode ser muito esparsa, levando-se em conta que nenhuma ERB cobre toda a região. Essa característica torna a implementação do problema mais próxima à realidade, principalmente em aplicações para as regiões de baixa demanda por serviços de comunicação ou projetos iniciais de atendimento.

Dependendo, então, da dimensão do problema, ou seja, considerando uma região com muitos pontos a serem cobertos e muitas ERB's candidatas, a obtenção de uma solução ótima ou até mesmo viável pode ficar comprometida se utilizarmos uma das modelagens descritas anteriormente.

Levando em conta esta dificuldade, apresentamos na seção seguinte uma nova metodologia de resolução do problema de localização de estações de rádio base. Tal metodologia é baseada nas técnicas de suavização (Polyak, 1988; Pillo et al., 1993 e Xavier, 2005) e penalização hiperbólica (Xavier, 2001).

\section{Modelagem Proposta}

Considere uma região em estudo para a qual se deve instalar um conjunto de estações de rádio base (ERB's), a fim de prover a cada ponto desta região um bom nível de cobertura (Mateus, 1998), associado a serviços de telefonia móvel / celular, e ao mesmo tempo, reduzir os custos de equipamento (minimizar o número de ERB's).

Do ponto de vista da nova modelagem proposta para este problema, definimos esta região a ser coberta por $S$, sendo $S \subset \mathfrak{R}^{2}$; por conseguinte, cada ponto a ser coberto é definido por $s$ tal que $s \in S$. Definimos, também, as coordenadas $x_{i} \in \mathfrak{R}^{2}(i=1, \ldots, q)$ que representam os centros dos círculos que cobrirão a região $S$. Estes centros estão associados aos pontos de localização das ERB's e serão obtidos no decorrer do processo de resolução do problema. Vale ressaltar que a medida dos raios dos círculos, também obtida na resolução do problema, representará o raio $z$ de cobertura de cada ERB. Esse raio é, por sua vez, função da potência de transmissão da antena (Mateus, 1998 e Nascimento, 2000). 
Observamos que essa modelagem tem um caráter de primeira aproximação para o problema de localização de ERB's e, ao mesmo tempo, um ponto de partida para as modelagens subseqüentes que propomos para este problema (Brito, 2004).

Fornecido, então, um ponto genérico $s$ da região $S$, devemos calcular inicialmente a distância deste ponto ao centro do círculo mais próximo, ou seja, qual a estação de rádio base que está mais próxima deste ponto.

$$
d(s, x)=\min _{i=1, \ldots, q}\left(\left\|s-x_{i}\right\|\right)
$$

A distância $d(s, x)$ fornece uma medida da cobertura de um ponto $s \in S$. Uma medida da qualidade da cobertura da região $S$ pelos $q$ círculos (ERB's) é dada pela maior distância $d(s, x)$, ou seja, qual ponto tem sua estação mais próxima situada a uma maior distância (cobertura mais crítica).

$$
D(x)=\max _{s \in S} d(\mathrm{~s}, \mathrm{x})
$$

A localização ótima das estações de rádio base deve representar a melhor qualidade de cobertura da região $S$, isto é, deve minimizar a cobertura mais crítica, de forma que não tenhamos pontos $s \in S$ descobertos.

$$
\min _{x} D(x)
$$

Após esta análise, temos então o seguinte problema:

$$
\min _{x} \max _{s \in S} \min _{i=1, \ldots, q}\left(\left\|S-x_{i}\right\|\right)
$$

\subsection{Resolução do problema}

Para que seja obtida a solução numérica de (4), devemos discretizar a região $S$ em um conjunto finito de pontos $s_{j}, j=1, \ldots, m$, que são os pontos da região que serão atendidos pelas ERB's. Desta maneira, obtemos então o seguinte problema:

$$
\min _{x} \max _{j=1, \ldots, m} \min _{i=1, \ldots, q}\left(\left\|s_{j}-x_{i}\right\|\right)
$$

Observamos que no nível mais interno de (5), para cada ponto $s_{j}, j=1, \ldots, m$ da região $S$, devemos resolver o subproblema:

$$
\min _{i=1, \ldots, q}\left(\left\|s_{j}-x_{i}\right\|\right)
$$

Denominamos por $z_{j}(x)$ a solução de (6) associada a um ponto $s_{j}$ :

$$
z_{j}(x)=\min _{i=1, \ldots, q}\left(\left\|S_{j}-x_{i}\right\|\right)
$$

Ou seja, para cada ponto $s_{j}$ da região $S$, deve-se definir a sua estação de rádio base associada mais próxima, com coordenadas $x_{i}$ e o seu raio de cobertura $z_{j}(x)$. 
Desta forma, $z_{j}(x)$ deve satisfazer necessariamente ao conjunto de desigualdades:

$$
z_{j}(x)-\left\|s_{j}-x_{i}\right\| \leq 0, \quad i=1, \ldots, q
$$

No nível intermediário de (5), temos o seguinte problema:

$$
\max _{j=1, \ldots, m} z_{j}(x)
$$

que corresponde à medida da pior cobertura associada ao posicionamento $x$ do conjunto de estações.

Considerando $z(x)$ como valor ótimo deste problema, temos:

$$
z(x) \geq z_{j}(x), \quad j=1, \ldots, m
$$

Observamos que no nível mais externo de (5) não há alteração se dissociamos $z$ e $x$. Desta forma, obtemos o seguinte problema equivalente:

$$
\begin{gathered}
\begin{array}{c}
\text { Minimizar } z \\
x, z
\end{array} \\
\text { Sujeito a }\left\{\begin{array}{l}
z_{j}=\min _{i=1, \ldots, q}\left\|s_{j}-x_{i}\right\|, j=1, \ldots, m \\
z \geq z_{j}, j=1, \ldots, m
\end{array}\right.
\end{gathered}
$$

Com base nas restrições de (8) e (11), definimos o seguinte problema:

$$
\begin{gathered}
\begin{array}{c}
\text { Minimizar } \\
x, z
\end{array} \\
\text { Sujeito a }\left\{\begin{array}{l}
z_{j}-\left\|s_{j}-x_{i}\right\| \leq 0, j=1, \ldots, m, i=1, \ldots, q \\
z \geq z_{j}, j=1, \ldots, m
\end{array}\right.
\end{gathered}
$$

Porém, devemos observar que o problema (12) não equivale ao problema (5), uma vez que as desigualdades (12.1) não garantem estritamente o cumprimento das igualdades (7). Além disso, como as variáveis $z_{j}$ estão livres inferiormente, pode-se verificar facilmente que a solução do problema (12) é ilimitada inferiormente.

Sendo assim, precisamos modificar o problema (12), de forma que seja considerada a observância estrita das restrições de igualdade (7).

Nesta parte do desenvolvimento, baseando-se no trabalho de Xavier (2005), introduzimos a seguinte função auxiliar:

$$
\varphi(x, \lambda)=\lambda . \text { máximo }\{0, x\}
$$

Sendo $\lambda \geq 1$, o parâmetro que representa a inclinação da função $\varphi($.$) .$

Se as desigualdades de (8) são válidas, então, necessariamente, deve ser observada a restrição:

$$
\sum_{i=1}^{q} \varphi\left(z_{j}-\left\|s_{j}-x_{i}\right\|, \lambda\right)=0, \quad j=1, \ldots, m .
$$


Com a substituição das desigualdades (12.1) por (14) no problema (12), obtemos um problema equivalente, que mantém a característica de ser ilimitado inferiormente. Ou seja, pela forma de definição da função objetivo e das restrições (14) e (12.2), ao resolvermos o problema (12) teremos $z<0$ e conseqüentemente $z_{j}<0, j=1, \ldots, m$, o que do ponto de vista da aplicação real que abordamos é inaceitável. Desta forma, para contornar este aspecto intrínseco ao problema (12) vamos definir o seguinte problema:

$$
\begin{gathered}
\qquad \begin{array}{c}
\text { Minimizar } z, z \\
\text { Sujeito a } \\
\sum_{i=1}^{q} \varphi\left(z_{j}-\min _{i=1, \ldots, q}\left\|s_{j}-x_{i}\right\|, \lambda\right)>0 \quad, j=1, \ldots, m \\
z \geq z_{j}, j=1, \ldots, m
\end{array}
\end{gathered}
$$

Ou, de uma forma alternativa, podemos perturbar a equação (15.1) e definir o problema:

$$
\begin{aligned}
& \underset{x, z}{\operatorname{Minimizar} \quad z} \\
& \text { Sujeito a }\left\{\begin{array}{l}
\sum_{i=1}^{q} \varphi\left(z_{j}-\min _{i=1, \ldots, q}\left\|s_{j}-x_{i}\right\|, \lambda\right) \geq \varepsilon>0 \quad, j=1, \ldots, m \\
z \geq z_{j}, j=1, \ldots, m
\end{array}\right.
\end{aligned}
$$

Poderíamos, também, gerar uma seqüência decrescente de valores $\left\{\varepsilon^{k}\right\}$ pela perturbação do parâmetro $\varepsilon$.

Desde que o problema (15) é o limite de (16) quando $\varepsilon \rightarrow 0_{+}$, podemos resolver o problema (15) através da resolução de uma seqüência de problemas do tipo (16) onde $\varepsilon=\varepsilon_{k} \rightarrow 0_{+}$.

No trabalho de Xavier (2005) é apresentado em detalhes um conjunto de resultados teóricos associados à resolução do problema (15). Estes resultados garantem a equivalência do problema (15) com o problema (11), no que concerne a solução ótima e a existência de pelo menos uma solução ótima (no problema (15)), onde todos os raios são iguais, ou seja, $z_{j}^{*}=z^{*}, j=1, \ldots, m$.

Com base nestes resultados, podemos reduzir consideravelmente a dimensão do problema, isto é, podemos definir o seguinte problema:

$$
\begin{gathered}
\text { Minimizar } z \\
\text { Sujeito a }\left\{\sum_{i=1}^{q} \varphi\left(z-\left\|s_{j}-x_{i}\right\|, \lambda\right)>0 \quad, j=1, \ldots, m\right.
\end{gathered}
$$

Devemos destacar que o problema (17) é definido num espaço de dimensão $(2 q+1)$, que é muito menor do que o espaço do problema (15), que tem dimensão $(2 q+m+1)$; isto é, no novo problema, o número de variáveis é da ordem do número de ERB's que se deseja instalar na região em estudo, e não mais do número de pontos de discretização da região $\mathrm{S}$, que serão cobertos pelas estações de rádio base. 
Em seu trabalho sobre recobrimento, Xavier (2005) também apresenta de forma detalhada um conjunto de resultados teóricos que garantem a equivalência entre os problemas (15) e (17). A apresentação destes resultados está fora do escopo deste trabalho.

Todavia, o problema (17) tem como espaço viável um conjunto aberto, inviabilizando a aplicação das condições de Karush, Khun e Tucker (Bazaraa et al., 1993). Ademais, possui uma estrutura muito rígida, não-diferenciável. Observando a definição da função $\varphi($.$) , temos$ que a mesma não é diferenciável nos pontos da forma $z=\left\|s_{j}-x_{i}\right\|$ e a própria função $\left\|s_{j}-x_{i}\right\|$ é não diferenciável nos pontos da forma $s_{j}=x_{i}$.

Para resolver numericamente o problema (17), ao invés dos métodos ortodoxos associados à programação não-diferenciável (LeMarechal, 1981), utilizamos a idéia de suavização apresentada no trabalho de Xavier (2005). Abaixo, temos as funções suavizadoras utilizadas na modelagem do problema (17).

$$
\begin{gathered}
\phi\left(y, \lambda, \tau_{1}\right)=\lambda\left(\frac{y+\sqrt{y^{2}+\tau_{1}^{2}}}{2}\right) \\
\theta\left(y, \tau_{2}\right)=\sqrt{y^{2}+\tau_{2}^{2}}
\end{gathered}
$$

A função $\phi($.$) definida acima constituirá uma aproximação (suavização) da função \varphi($ ) e a função $\theta\left(\right.$.) constituirá uma aproximação da função $\left\|s_{j}-x_{i}\right\|$ (considerando $y=\left\|s_{j}-x_{i}\right\|$ ), sendo $\tau_{1}$ e $\tau_{2}$ os parâmetros de suavização.

A seguir, apresentamos as propriedades das funções $\phi($.$) e \theta($.

(1) $\lim _{\tau_{1} \rightarrow 0} \phi\left(y, \lambda, \tau_{1}\right)=\left\{\begin{array}{c}0 \text { se } y \leq 0 \\ y \text { se } y>0,(\lambda=1)\end{array}\right\} \quad \lim _{\tau_{2} \rightarrow 0} \theta\left(y, \tau_{2}\right)=\left\{\begin{array}{c}y \text { se } y \geq 0 \\ -y \text { se } y<0\end{array}\right\}$

(2) $\phi($.$) e \theta($.$) são continuamente diferenciáveis em y$.

A propriedade (1), observada para as duas funções suavizadoras, mostra que a diferença entre $\phi($.$) e \varphi($.$) ou entre \theta($.$) e \left\|s_{j}-x_{i}\right\|$, pode ser feita tão pequena quanto se desejar, através da manipulação dos parâmetros $\tau_{1}$ e $\tau_{2}$. Estes parâmetros introduzidos nos modelos de suavização estão associados ao desvio máximo entre as funções $\phi($.$) e \varphi($.$) e entre as$ funções $\theta\left(\right.$.) e $\left\|s_{j}-x_{i}\right\|$. Além disso, como $\tau_{1}$ e $\tau_{2}$ são parâmetros externos, é possível controlar totalmente o nível de desvio produzido pela suavização.

A propriedade (2), referente à diferenciabilidade infinita, permitirá o uso de algoritmos de minimização mais robustos e eficientes que utilizam as informações das derivadas primeira e segunda.

Utilizando as funções $\phi($.$) e \theta($.$) e a modelagem alternativa (16), substituímos a resolução$ de (17) pela resolução de uma seqüência de problemas (20), abaixo, que se aproximam gradativamente do problema (17), através da redução dos parâmetros $\tau_{1}, \tau_{2}$ e $\varepsilon$. E além disso, o problema (20) não mais apresenta os dois aspectos complicadores destacados para o problema (17): 


$$
\begin{gathered}
\underset{x, z}{\text { Minimizar }} z \\
\text { Sujeito a }\left\{\sum_{i=1}^{q} \phi\left(z-\theta\left(\left\|s_{j}-x_{i}\right\|, \tau_{2}\right), \lambda, \tau_{1}\right) \geq \varepsilon \quad, j=1, \ldots, m\right.
\end{gathered}
$$

sendo $s_{j}=\left(s_{j}^{1}, s_{j}^{2}\right)$ os pontos a serem atendidos pelas estações e $x_{i}=\left(x_{i}^{1}, x_{i}^{2}\right)$ os seus respectivos centros. Podemos, então, reescrever (20) da seguinte forma:

$$
\begin{gathered}
\sum_{i=1}^{q} \frac{\lambda}{2}\left(\mathrm{z}-\sqrt{\sum_{\mathrm{k}=1}^{2}\left(x_{i}^{k}-s_{j}^{k}\right)^{2}+\tau_{2}^{2}}+\sqrt{\left[\mathrm{z}-\sqrt{\left.\sum_{\mathrm{k}=1}^{2}\left(x_{i}^{k}-s_{j}^{k}\right)^{2}+\tau_{2}\right]^{2}}+\tau_{1}^{2}\right)} \geq \varepsilon\right. \\
j=1, \ldots, m
\end{gathered}
$$

A seguir, apresentamos o algoritmo desenvolvido para resolução de (21).

\section{ALGORITMO DE SUAVIZAÇ̃̃O HIPERBÓLICA}

21) Faça $\mathrm{k}=0$ e defina valores iniciais para $z^{0}, x^{0}, \lambda, \tau_{1}^{1}, \tau_{2}^{1}, \varepsilon^{1}, \delta$

22) Defina valores para $\rho_{1}, \rho_{2}$ tais que $0<\rho_{2} \leq \rho_{1}<1$

23) Repita

24) Faça $k=k+1$

25) Defina os valores de $d$ e $\alpha$ (parâmetros de penalização hiperbólica)

26) Resolva o problema (21) através da penalização hiperbólica, com $x^{k-1}$ e $z^{k-1}$

27) Faça $\tau_{1}^{k+1}=\rho_{1} \cdot \tau_{1}^{k} \quad$ Faça $\tau_{2}^{k+1}=\rho_{1} \cdot \tau_{2}^{k}$

28) $\quad$ Faça $\varepsilon^{k+1}=\rho_{2} \cdot \varepsilon^{k}$

29) Até $\left(\left|z^{k+1}-z^{k}\right| \leq \delta\right) \quad$ (Fim - Repita)

30) Fim.

Essencialmente, no algoritmo apresentado acima, é resolvida uma seqüência de problemas de programação não-linear restritos no passo 6, através do algoritmo de penalização hiperbólica, apresentado no trabalho de Xavier (2001). No primeiro e segundo passos do algoritmo, definimos valores iniciais para a solução (raios e centros) e para os parâmetros de suavização e de tolerância.

No passo 9, temos a condição de parada do algoritmo de suavização hiperbólica associada a redução do raio de cobertura. No passo 5 , definimos os parâmetros que serão utilizados na penalização. Nos passos 7 e 8 efetuamos a redução gradativa dos parâmetros $\tau_{1}, \tau_{2}$ e $\varepsilon$. 


\section{Modelagens Variantes}

Nesta seção, apresentamos três novas modelagens que foram motivadas pelo estudo das características do problema de localização de ERB's e na modelagem min-max-min básica.

A resolução numérica da modelagem min-max-min básica fornece os valores de $x_{i} \mathrm{e} z$. Os valores $x_{i}$, conforme já abordado, representam os pontos de localização das ERB's e a variável $z$ representa o raio de cobertura de cada uma das ERB's. Este raio está normalmente associado ao alcance e à qualidade de recepção dos sinais emitidos pelas antenas (potência do sinal) que compõem cada ERB. Devemos observar que há uma relação entre o número de estações e o raio de cobertura $z$ de cada uma das estações. Com o aumento do parâmetro $q$ (número de ERB's), há uma redução do tamanho do raio de cobertura $z$ de cada estação e vice-versa.

Se o valor $z$ obtido para o raio de cobertura não estiver adequado às necessidades de serviço da região, o valor inicial do parâmetro $q$ pode ser aumentado ou reduzido, até que seja atingido um patamar pré-estabelecido de qualidade de cobertura. Esse procedimento pode ser facilmente efetuado através de uma busca binária no valor de $q$, de forma que o valor de $z$ respeite os limites de cobertura associados ao problema real ( $\left.\mathrm{z}_{\text {mínimo }} \leq z \leq \mathrm{z}_{\text {máximo }}\right)$.

Observamos que esta modelagem inicial dá uma visão geral da distribuição das estações de rádio base sobre a região $S$. Todavia, com intuito de tornar a solução desta problemática mais aderente a realidade, isto é, considerando as características intrínsecas ao problema de localização ERB's, numa dada região $S$, tais como: a diversidade na propagação e alcance dos sinais de rádio, a distribuição dos usuários, os obstáculos decorrentes do relevo, topografia e a presença de objetos (construções, transmissores, etc), apresentamos três modelagens variantes (Brito, 2004).

Nas modelagens, apresentadas a seguir, encapsulamos o aspecto da distância máxima entre as estações, priorizamos pontos candidatos à instalação das ERB's e definimos agrupamentos de locais candidatos para instalação de ERB's.

Com a utilização dessas modelagens ou de uma combinação das mesmas, podemos ter uma idéia bem melhor para a distribuição do sistema como um todo.

\subsection{Modelagem M1: estações com localização pré-definida}

Nesta primeira modelagem, será contemplada a situação em que algumas das estações têm a localização pré-definida. Dessa forma, considere que para a região $S$ a ser coberta, escolhamos um conjunto $y_{r}=\left(y_{r}^{1}, y_{r}^{2}\right)$ de pontos $(r=1, \ldots, \bar{q} \leq q)$. Ou seja, vamos escolher alguns pontos $y_{r}$ que estão na região $S$ e dos quais as estações de rádio base deverão estar a uma certa distância pré-definida. Isto é, as estações deverão estar localizadas (instaladas) numa vizinhança dos pontos $y_{r}$ (Figura 2).

Normalmente, a escolha desses pontos (locais) é feita $a d-h o c$ pela área de planejamento da companhia e está diretamente correlacionada às análises de propagação de sinal, ao relevo da região, aos tipos de construção, à densidade de usuários e a alguns obstáculos, conforme descrito por Mateus (1998). 
Na Figura 2, apresentamos um exemplo com $q=4, \bar{q}=3$, isto é, temos três estações que deverão estar numa vizinhança de especificados pontos $y_{1}, y_{2}, y_{3}$ e uma estação com posicionamento livre.

Associemos a cada ponto $y_{r}=\left(y_{r}^{1}, y_{r}^{2}\right)$, definido para esta região, um ponto $x_{r}=\left(x_{r}^{1}, x_{r}^{2}\right)$ que representará o centro de uma das $\bar{q}$ estações, de tal forma que a distância entre cada ponto $y_{r}$ e o seu respectivo centro $x_{r}$ seja maior ou igual que a um valor $d_{r}$ e menor ou igual a um valor $D_{r}$, ambos definidos a priori.

A racionalidade de se definir que os centros das estações estejam numa vizinhança dos pontos $y_{r}$ é a de garantir que essas estações estejam a uma distância máxima de $D_{r}$ de: hospitais, grandes centros de informação, centrais de emergência, etc, que também estão em uma vizinhança dos pontos $y_{r}$.

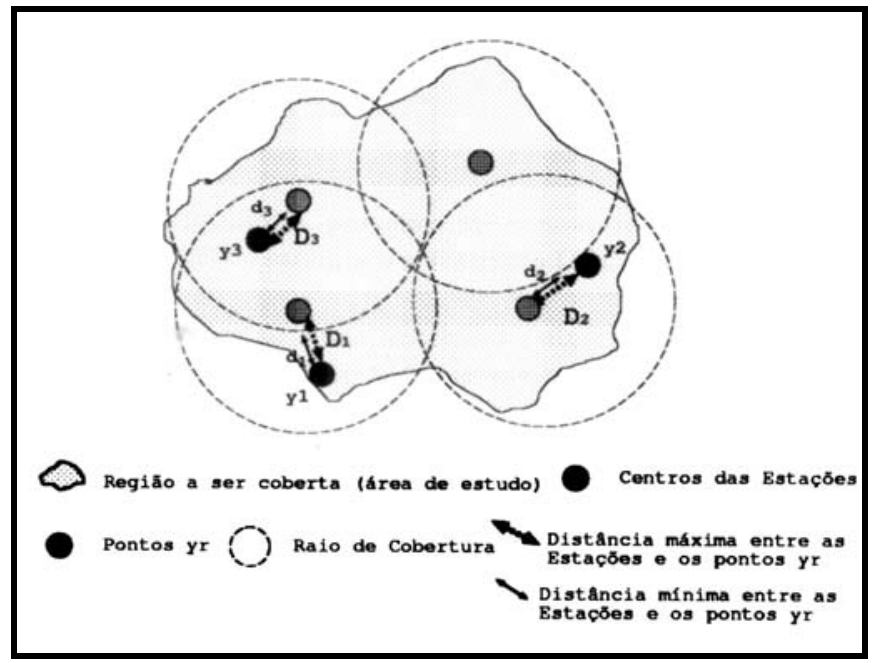

Figura 2 - Exemplo da Modelagem M1.

Todavia, além da existência desses pontos de demanda, podemos ter obstáculos como transmissores, construções, etc., também próximos de $y_{r}$. A fim de garantir que esses obstáculos não interfiram na comunicação para pontos de demanda próximos de $y_{r}$, definimos uma distância $d_{r}$, como a medida de distância mínima (afastamento mínimo) que a estação deve estar do ponto $y_{r}$, evitando a interferência e conseqüentemente a perda de comunicação.

Assim, do ponto de vista de modelagem, definidos os valores $D_{r}$ e $d_{r}$ e os seus respectivos pontos $y_{r},(r=1, \ldots, \bar{q})$, devemos estabelecer que:

$$
\begin{aligned}
& \left\|y_{r}-x_{r}\right\| \leq D_{r}, \quad r=1, \ldots, \bar{q} \leq q \\
& \left\|y_{r}-x_{r}\right\| \geq d_{r}, \quad r=1, \ldots, \bar{q} \leq q
\end{aligned}
$$


Considerando então estas novas restrições, temos a seguinte modelagem:

$$
\begin{gathered}
\text { Minimizar } \\
x, z \\
\sum_{i=1}^{q} \phi\left(z-\theta\left(\left\|s_{j}-x_{i}\right\|, \tau_{2}\right), \lambda, \tau_{1}\right) \geq \varepsilon \quad, \quad j=1, \ldots, m \\
\left(y_{r}^{1}-x_{r}^{1}\right)^{2}+\left(y_{r}^{2}-x_{r}^{2}\right)^{2} \leq D_{r}{ }^{2}, \quad r=1, \ldots, \bar{q} \leq q \\
\left(y_{r}^{1}-x_{r}^{1}\right)^{2}+\left(y_{r}^{2}-x_{r}^{2}\right)^{2} \geq d_{r}{ }^{2}, \quad r=1, \ldots, \bar{q} \leq q
\end{gathered}
$$

Observamos que as restrições (24.1) garantem que todos os pontos $s_{j} \in S$ serão cobertos por alguma estação de rádio base e as restrições (24.2) e (24.3) garantem que cada uma das estações de centro $x_{r}$ estarão numa vizinhança de $y_{r}$. As restrições (24.2) e (24.3) são equivalentes às restrições (22) e (23) e tornam a resolução da modelagem M1 mais simples.

\subsection{Modelagem M2: agrupamento de estações}

Nesta segunda modelagem alternativa, vamos considerar que as $q$ estações de rádio base, sejam distribuídas em $n$ agrupamentos disjuntos. Ou seja, se $Q$ é o conjunto de todas as estações, temos: $Q=Q_{1} \cup Q_{2} \cup \ldots \cup Q_{n}$. Sendo $\left|Q_{r}\right|, r=1, \ldots, n$, o número de estações de rádio base em cada agrupamento.

$\mathrm{Na}$ Figura 3 apresentamos um exemplo desta modelagem, considerando $n=3$ e $\left|Q_{1}\right|=3,\left|Q_{2}\right|=4,\left|Q_{3}\right|=2$.

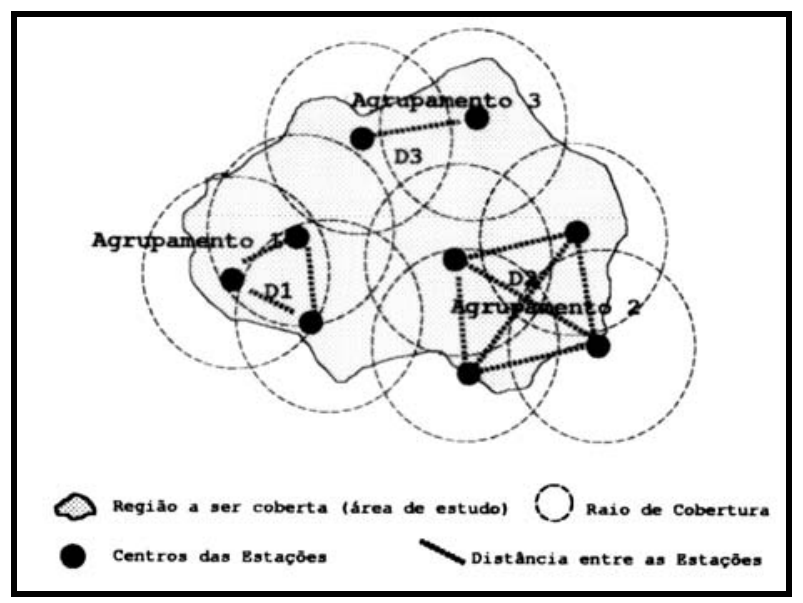

Figura 3 - Exemplo da modelagem M2.

Para cada agrupamento $Q_{r},(r=1, \ldots, n)$, definimos uma medida $D_{r}$, que representa a distância máxima entre os centros das estações duas a duas. 
A racionalidade da formação de agrupamentos de estações de rádio base com distâncias diferenciadas está associada às questões de utilização do espectro de freqüências e à densidade de usuários.

Conforme descrito nos trabalhos de Mateus (1998) e Nascimento (2000), o espectro de freqüências disponível para o serviço de telefonia celular é limitado. E, além disso, em áreas urbanas, há uma grande concentração de usuários do serviço de telefonia. Dessa forma, o espectro de frequências deve ser utilizado de uma forma parcimoniosa.

Para atender às demandas dos grandes centros urbanos, podemos, então, formar mais agrupamentos de estaçoes de rádio base, colocando estas estações mais próximas uma das outras e disponibilizar, para cada um destes agrupamentos, um conjunto de canais de freqüência menor e que poderá ser reaproveitado para outros agrupamentos vizinhos sem que haja interferência de canais (Mateus, 1998) entre as estações e de forma que todos os usuários sejam atendidos.

Já para áreas pouco povoadas, áreas rurais, etc, podemos definir um número menor de agrupamentos com as estações mais distanciadas, tendo em vista que o número de usuários é bem menor.

Deseja-se então, que as $q$ estações sejam distribuídas em $n$ agrupamentos, tal que para estações de centros $x_{l}=\left(x_{l}^{1}, x_{l}^{2}\right)$ e $x_{u}=\left(x_{u}^{1}, x_{u}^{2}\right)$ pertencentes a um mesmo agrupamento tenhamos:

$$
\begin{gathered}
\left\|x_{l}-x_{u}\right\|_{2} \leq D_{1}, \quad l=1, \ldots,\left|Q_{1}\right|-1, \quad u=l+1, \ldots,\left|Q_{1}\right| \\
\quad(\text { primeiro agrupamento }) \\
\left\|x_{l}-x_{u}\right\|_{2} \leq D_{p}, \quad l=\left(\sum_{r=1}^{p-1}\left|Q_{r}\right|\right)+1, \ldots,\left(\sum_{r=1}^{p}\left|Q_{r}\right|\right)-1, \quad u=l+1, \ldots, \sum_{r=1}^{p}\left|Q_{r}\right| \\
(\text {-ésimo agrupamento }) \quad(1<p \leq n)
\end{gathered}
$$

Considerando então estas novas restrições, temos a seguinte modelagem:

$$
\begin{gathered}
\begin{array}{c}
\text { Minimizar } \\
x, z
\end{array} \\
\sum_{i=1}^{q} \phi\left(z-\theta\left(\left\|s_{j}-x_{i}\right\|, \tau_{2}\right), \lambda, \tau_{1}\right) \geq \varepsilon \quad, j=1, \ldots, m \\
\left(x_{l}^{1}-x_{u}^{1}\right)^{2}+\left(x_{l}^{2}-x_{u}^{2}\right)^{2} \leq D_{1}^{2}, \quad l=1, \ldots,\left|Q_{1}\right|-1, \quad u=l+1, \ldots,\left|Q_{1}\right| \\
(\text { primeiro agrupamento }) \\
\left(x_{l}^{1}-x_{u}^{1}\right)^{2}+\left(x_{l}^{2}-x_{u}^{2}\right)^{2} \leq D_{p}{ }^{2}, l=\left(\sum_{r=1}^{p-1}\left|Q_{r}\right|\right)+1, \ldots,\left(\sum_{r=1}^{p}\left|Q_{r}\right|\right)-1, u=l+1, \ldots, \sum_{r=1}^{p}\left|Q_{r}\right| \\
(\text {-ésimo agrupamento }) \quad(1<p \leq n)
\end{gathered}
$$

Nesta modelagem, as restrições (26.1) garantem que todos os pontos $s_{j} \in S$ serão cobertos por alguma estação de rádio base e as restrições (26.2) definem as distâncias máximas entre as estações que estão em um mesmo agrupamento. As restrições do tipo (26.2) são equivalentes às restrições do tipo (25) e tornam a resolução da modelagem $\mathbf{M 2}$ mais simples. 
Observamos que, a despeito das restrições (26.2), o problema será sempre viável, independente dos valores que venha a se definir para o parâmetro $D_{r}$, ou seja, a redução de $D_{r}$ será compensada pelo aumento do raio $z$ e vice-versa.

\subsection{Modelagem M3: conjunto de pontos candidatos para a instalação de ERB's}

Nesta modelagem, vamos considerar que sejam definidos a priori $q$ conjuntos de pontos candidatos à instalação das estações de rádio base (Figura 4). Cada um destes conjuntos será designado por $H_{r}, \quad r=1, \ldots, q$ contendo $\left|H_{r}\right|$ pontos candidatos $h_{r l}=\left(h_{r l}^{1}, h_{r l}^{2}\right)$, $l=1, \ldots,\left|H_{r}\right|, \cap_{r=1}^{q} H_{r}=\{\}$.

Conforme descrito em Mateus (1998), os pontos $h_{r l}$ associados a cada um dos conjuntos $H_{r}$ são definidos a priori pela área de planejamento da companhia prestadora de serviços através de análises de propagação de sinal, relevo, etc.

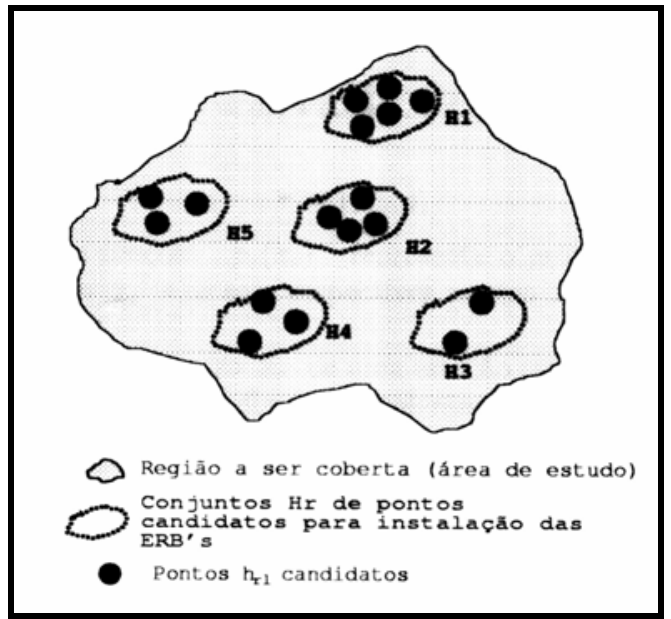

Figura 4 - Exemplo da modelagem M3.

Desta forma, vamos considerar que, para cada um dos conjuntos $H_{r}, r=1, \ldots, q$, será selecionado apenas um ponto $h_{r l}$ para instalação das ERB's. Ou seja, teremos então, um total de $q$ pontos associados às ERB's de centros $\left(x_{1}, x_{2}, \ldots, x_{q}\right)$.

Para cada ponto $h_{r l} \in H_{r} \quad r=1, \ldots, q, \quad l=1, \ldots,\left|H_{r}\right|$, podemos definir, então, uma função que representará a distância entre cada ponto $h_{r l}$ e suas respectivas estações:

$$
\bar{D}_{r l}=\sqrt{\left(x_{r}^{1}-h_{r l}^{1}\right)^{2}+\left(x_{r}^{2}-h_{r l}^{2}\right)^{2}}, \quad r=1, \ldots, q, \quad l=1, \ldots,\left|H_{r}\right|
$$

Ou de forma equivalente, podemos trabalhar com:

$$
\bar{d}_{r l}=\bar{D}_{r l}^{2}=\left(x_{r}^{1}-h_{r l}^{1}\right)^{2}+\left(x_{r}^{2}-h_{r l}^{2}\right)^{2}, \quad r=1, \ldots, q, \quad l=1, \ldots,\left|H_{r}\right|
$$


Como para cada conjunto $H_{r}$ deve-se selecionar apenas um ponto $h_{r l}$, então, definido um número $q$ de estações, devemos ter pontos $h_{r l}$ e, conseqüentemente, centros $x_{r}=\left(x_{r}^{1}, x_{r}^{2}\right)$, tais que $\bar{d}_{r l}=0, r=1, \ldots, q$.

Para auxiliar a apresentação do problema, vamos definir a seguinte função produto intra-grupo:

$$
p_{r}\left(x_{r}\right)=\bar{d}_{r 1} * \bar{d}_{r 2} * \ldots * \bar{d}_{r\left|H_{r}\right|}, \quad r=1, \ldots, q
$$

ou seja, cada uma das funções $p_{r}\left(x_{r}\right)$ representa o produto das distâncias entre uma estação de centro $x_{r}$ e os seus respectivos pontos $h_{r l} \in H_{r}$.

A fim de garantir que o centro $x_{r}$ de uma estação estará associado a apenas um dos pontos de $H_{r}$, podemos definir uma tolerância $\delta_{r} \geq 0$ convenientemente para cada grupo e as seguintes restrições:

$$
p_{r}\left(x_{r}\right) \leq \delta_{r}, \quad r=1, \ldots, q
$$

Considerando essas restrições e as restrições de cobertura da modelagem min-max-min básica temos então a seguinte modelagem:

$$
\begin{gathered}
\begin{array}{c}
\text { Minimizar } \\
x, z
\end{array} \\
\sum_{i=1}^{q} \phi\left(z-\theta\left(\left\|s_{j}-x_{i}\right\|, \tau_{2}\right), \lambda, \tau_{1}\right) \geq \varepsilon, \quad j=1, \ldots, m \\
\prod_{l=1}^{\left|H_{r}\right|} \bar{d}_{r l} \leq \delta_{r}, \quad r=1, \ldots, q
\end{gathered}
$$

Observamos que os parâmetros $\delta_{r}$ são definidos de forma conveniente, levando em conta o número de pontos candidatos em cada conjunto $H_{r}$ e a escala de representação destes pontos na região $S$.

As restrições (31.1) garantem a cobertura total da região $S$ e as restrições (31.2) garantem que em cada conjunto $H_{r}$ será selecionado apenas um ponto $h_{r l}$.

Devemos ainda ressaltar, que esta modelagem pode ser convenientemente manipulada de forma a convergir para as modelagens de programação inteira ortodoxas, que são utilizadas para o problema de localização de ERB's, sem o uso explícito de variáveis $(0,1)$ (Brito, 2004).

\section{Resultados Computacionais}

Nesta seção, faremos uma exposição concisa dos resultados numéricos/computacionais obtidos para a modelagem min-max-min básica (modelagem M0) e para as modelagens variantes apresentadas na seção anterior.

Os resultados que serão apresentados nas tabelas a seguir foram obtidos a partir do desenvolvimento de dois programas elaborados em linguagem de programação Delphi $6.0 \mathrm{e}$ que foram executados num microcomputador IBM-PC dotado de um processador Pentium IV (1.6 GHZ) com $256 \mathrm{Mb}$ de memória. 
O primeiro programa gera para o interior da poligonal (região $S$ ), uma malha, ou conjunto de pontos eqüidistantes, que pode ter uma maior ou menor densidade (subdivisões), com cada ponto $s_{j}$ dessa malha representando um conjunto de usuários que devem ser atendidos pelas estações de rádio base. Alternativamente, pode-se também gerar os pontos no interior da poligonal aleatoriamente, isto é, sem que os pontos $s_{j}$ estejam eqüidistantes (caso da malha).

Completando o conjunto de dados de entrada do programa, é solicitado ao usuário o número $q$ de ERB's (círculos) que serão utilizados para cobrir a região $S$. De posse desse número, o programa gera de forma aleatória os centros (coordenadas) $x_{i},(i=1, \ldots, q)$, das ERB's, exigindo apenas que estes centros $x_{i}=\left(x_{i}^{1}, x_{i}^{2}\right)$ pertençam ao interior da região $S$. E, finalmente, define-se um raio inicial $z^{0}$ associado a cada centro $x_{i}$ tal que $z^{0}=\operatorname{diam}(S)$, onde $\operatorname{diam}(S)$ representa o diâmetro da região $S$ (Lages, 1991).

Considerando então, todas essas informações, é gerado um arquivo de saída que contém os pontos $s_{j}=\left(s_{j}^{1}, s_{j}^{2}\right)$ delimitados pela poligonal, o número de ERB's necessárias para atender a demanda da região $S$, as coordenadas iniciais $x_{i}, i=1, \ldots, q$ das ERB's e o seu raio de cobertura $z^{0}$.

Ainda utilizando o programa de geração de dados, é possível considerar, para o interior da poligonal (região $S$ ), os seguintes parâmetros associados às modelagens variantes:

Tabela 1 - Parâmetros considerados no programa de geração de dados.

\begin{tabular}{|c|c|}
\hline Mogelagem & Parâmetros \\
\hline $\begin{array}{l}\text { M1: Estações com Localização } \\
\text { Pré-Definida }\end{array}$ & $\begin{array}{l}\text { - Pontos } y_{r}, r=1, \ldots, \bar{q} \leq q \text {, dos quais a ERB ficará próxima } \\
\text { - Distância máxima } D_{r} \text { de uma ERB ao ponto } y_{r} \\
\text { - Distância mínima } d_{r} \text { de uma ERB ao ponto } y_{r}\end{array}$ \\
\hline M2: Agrupamento de Estações & $\begin{array}{l}\text { - Número de agrupamentos } n \\
\text { - Número de estações por agrupamento } \\
\text { - Distância } D_{r} \text { entre estações de cada agrupamento }\end{array}$ \\
\hline $\begin{array}{l}\text { M3: Conjunto de Pontos } \\
\text { Candidatos para Instalação de } \\
\text { ERB's }\end{array}$ & $\begin{array}{l}\text { - Número de conjuntos } H_{r}, \quad r=1, \ldots, q \text { com pontos } \\
\text { candidatos } \\
\text { - Pontos } h_{r l}, 1=1, \ldots,\left|H_{r}\right| \text { em cada conjunto } H_{r} \\
\text { - Tolerância } \delta_{r}\end{array}$ \\
\hline
\end{tabular}

Observamos que, neste trabalho, não foi considerada uma particular unidade de medida para os parâmetros de distância e localização $\left(D_{r}, d_{r}\right.$ e $\left.y_{r}\right)$ da Tabela 1 ou para o raio de cobertura $z$ e centros $x_{i}$ das estações. Ou seja, pode-se estabelecer uma escala considerando a distância em metros ou até mesmo em quilômetros, dependendo da região (associada ao problema real) que estiver sendo estudada. 
Para a implementação dos algoritmos associados à modelagem básica e às modelagens variantes, apresentadas neste trabalho, fizemos o uso de uma biblioteca de rotinas de programação não-linear, disponibilizada na internet por Debord (2000).

Em seguida, baseados no trabalho de Dennis \& Schnabel (1994), efetuamos adaptações em alguns procedimentos presentes nessa biblioteca, com a finalidade de utilizá-los no algoritmo de suavização hiperbólica. Nesse algoritmo, temos um conjunto de funções associadas aos processos de suavização e de penalização hiperbólica e ao cálculo dos gradientes.

Os dados de entrada comuns fornecidos para esses algoritmos foram: os pontos $s_{j}$ contidos na região $S$, o raio de cobertura e os centros iniciais das estações de rádio base, os parâmetros de suavização $\tau_{1}, \tau_{2}, \varepsilon, \rho_{1}, \rho_{2}$ e $\lambda$ e os parâmetros de penalização $d$ e $\alpha$.

Além desses parâmetros, foram fornecidos os parâmetros particulares de cada uma das modelagens variantes, apresentados na Tabela 1 . Na Tabela 2, temos os valores iniciais dos parâmetros utilizados no algoritmo de suavização hiperbólica apresentado na seção 3 .

Tabela 2 - Parâmetros utilizados no algoritmo de suavização hiperbólica.

\begin{tabular}{|c|c|}
\hline Parâmetro & Valor \\
\hline$\tau_{1}$ & 1 \\
\hline$\tau_{2}$ & 1 \\
\hline$\varepsilon$ & 1 \\
\hline$\rho_{1}, \rho_{2}$ & 0.5 \\
\hline$\lambda$ & 1 \\
\hline$d$ & 10 \\
\hline$\alpha$ & $\pi / 3$ \\
\hline
\end{tabular}

A seguir, apresentamos as tabelas com resultados obtidos para as quatro modelagens propostas neste trabalho, considerando um conjunto de problemas teste.

Obs.: com a finalidade de possibilitar a reprodução dos experimentos numéricos e a possível comparação com outros algoritmos, os dados utilizados nos problemas teste, apresentados nas tabelas a seguir, foram disponibilizados na página pessoal de um dos autores e podem ser obtidos na página http://paginas.terra.com.br/educacao/BRITOM/Dados_Recobrimento ou podem ser solicitados através dos e-mails dos autores. Nesta página de Internet, temos os arquivos com os dados e um texto explicativo sobre o layout destes arquivos.

\subsection{Resultados da modelagem M0 - cobertura total}

$\mathrm{Na}$ Tabela 3, apresentada a seguir, temos segundo a ordem das colunas: o nome do problema teste, o número de ERB's, o número de pontos $s_{j} \in S$ cobertos pelas ERB's, o raio de cobertura das ERB's, o total de iterações do método BFGS e o tempo de processamento, em segundos, gasto para resolver o problema. 
Pela Tabela 3 e pelas Figuras 5 e 6 (apresentadas a seguir), podemos observar que a modelagem min-max-min básica mostrou-se eficiente para problemas teste com um razoável número de variáveis e restrições.

Tabela 3 - Informações gerais sobre os problemas teste.

\begin{tabular}{|c|c|c|c|c|c|}
\hline Problema & No de ERB's & No de Pontos & Raio z & Iterações BFGS & $\begin{array}{c}\text { Tempo } \\
\text { CPU(Seg) }\end{array}$ \\
\hline AF180* & 17 & 16814 & 65.2308 & 360 & 3530 \\
\hline BH120* & 86 & 7301 & 26.7952 & 453 & 27900 \\
\hline BH150* & 30 & 11328 & 48.4354 & 409 & 6275 \\
\hline BH420* & 4 & 87776 & 144.0751 & 413 & 3323 \\
\hline RJ100* & 50 & 4668 & 36.0037 & 379 & 5583 \\
\hline RJ200* & 17 & 18341 & 96.5032 & 398 & 1580 \\
\hline AL10000** & 5 & 10096 & 146.2957 & 296 & 358 \\
\hline AL30000** & 3 & 30097 & 196.7092 & 335 & 731 \\
\hline SG4000** & 4 & 4125 & 146.6143 & 366 & 164 \\
\hline SG6000** & 7 & 6125 & 104.0437 & 329 & 350 \\
\hline
\end{tabular}

*Pontos eqüidistantes **Pontos distribuídos aleatoriamente

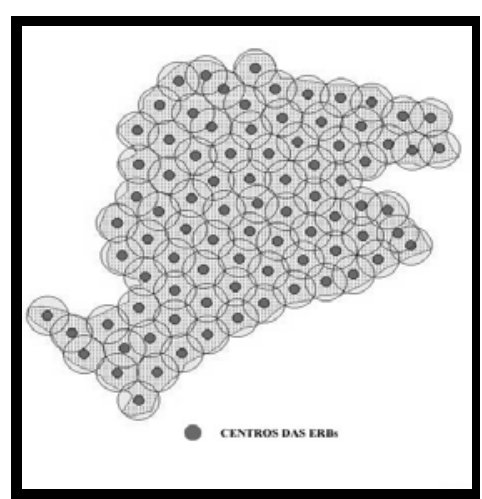

Figura 5 - Problema BH120.

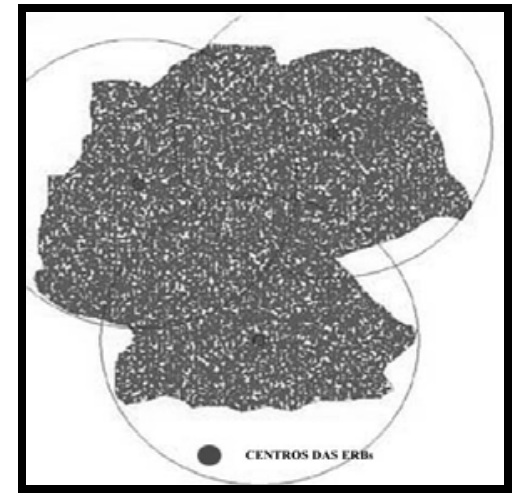

Figura 6 - Problema AL30000.

\subsection{Resultados da modelagem M1 - estações com localização pré-definida}

Analisando as Tabelas 4 e 5, segundo o critério do número de variáveis, de restrições e do número de estações com a localização pré-definida $\bar{q}$, podemos observar, novamente, que foi possível resolver problemas de diferentes dimensões. Ademais, verificamos (pela última coluna da Tabela 5) que as restrições de vizinhança foram satisfeitas para todos os problemas teste. 
Tabela 4 - Informações gerais sobre os problemas teste.

\begin{tabular}{|c|c|c|c|c|c|}
\hline Problema & $\mathbf{N}^{\mathbf{0}}$ de ERB's & $\mathbf{N}^{\mathbf{0}}$ de Pontos & Raio z & Iterações BFGS & $\begin{array}{c}\text { Tempo } \\
\text { CPU(Seg) }\end{array}$ \\
\hline FR70* & 5 & 3041 & 157.0017 & 95 & 50 \\
\hline FR100* & 10 & 6077 & 99.3580 & 414 & 702 \\
\hline FR140* & 7 & 11811 & 124.9341 & 379 & 833 \\
\hline FR150* & 4 & 13256 & 201.2486 & 302 & 447 \\
\hline FR4000** & 3 & 4131 & 216.5976 & 219 & 105 \\
\hline FR14000** & 6 & 14131 & 119.9159 & 387 & 844 \\
\hline
\end{tabular}

*Pontos eqüidistantes **Pontos distribuídos aleatoriamente

Tabela 5 - Distâncias máximas, mínimas e distâncias finais.

\begin{tabular}{|c|c|c|c|c|}
\hline Problema & $\bar{q}$ & $\begin{array}{c}\text { Distância Máxima } \\
D_{r}\end{array}$ & $\begin{array}{c}\text { Distância Mínima } \\
d_{r}\end{array}$ & Distâncias Finais $\left\|y_{r}-x_{r}\right\|=d_{l f}$ \\
\hline FR70* & 5 & $\begin{array}{l}\mathrm{D}_{1}=210 \mathrm{D}_{2}=210 \mathrm{D}_{3}=180 \\
\mathrm{D}_{4}=300 \mathrm{D}_{5}=200\end{array}$ & $\begin{array}{l}\mathrm{d}_{1}=70 \quad \mathrm{~d}_{2}=70 \quad \mathrm{~d}_{3}=70 \\
\mathrm{~d}_{4}=100 \mathrm{~d}_{5}=100\end{array}$ & $\begin{array}{ll}\mathrm{d}_{1 \mathrm{f}}=86.20 & \mathrm{~d}_{2 \mathrm{f}}=139.41 \\
\mathrm{~d}_{3 \mathrm{f}}=173.01 \mathrm{~d}_{4 \mathrm{f}}=192.50 \mathrm{~d}_{5 \mathrm{f}}=199.99\end{array}$ \\
\hline FR100* & 5 & $\begin{array}{l}\mathrm{D}_{1}=80 \mathrm{D}_{2}=90 \mathrm{D}_{3}=120 \\
\mathrm{D}_{4}=200 \mathrm{D}_{5}=60\end{array}$ & $\begin{array}{lll}\mathrm{d}_{1}=20 & \mathrm{~d}_{2}=30 & \mathrm{~d}_{3}=50 \\
\mathrm{~d}_{4}=10 & \mathrm{~d}_{5}=20\end{array}$ & $\begin{array}{ll}\mathrm{d}_{1 \mathrm{f}}=40.72 & \mathrm{~d}_{2 \mathrm{f}}=57.12 \\
\mathrm{~d}_{3 \mathrm{f}}=74.42 & \mathrm{~d}_{4 \mathrm{f}}=140.58 \quad \mathrm{~d}_{5 \mathrm{f}}=52.23\end{array}$ \\
\hline FR140* & 3 & $\mathrm{D}_{1}=30 \mathrm{D}_{2}=180 \mathrm{D}_{3}=240$ & $\mathrm{~d}_{1}=10 \quad \mathrm{~d}_{2}=20 \quad \mathrm{~d}_{3}=40$ & $\mathrm{~d}_{1 \mathrm{f}}=11.12 \mathrm{~d}_{2 \mathrm{f}}=118.60 \mathrm{~d}_{3 \mathrm{f}}=149.19$ \\
\hline FR150* & 1 & $\mathrm{D}_{1}=120$ & $\mathrm{~d}_{1}=10$ & $\mathrm{~d}_{1 \mathrm{f}}=86.00$ \\
\hline FR4000** & 1 & $\mathrm{D}_{1}=90$ & $\mathrm{~d}_{1}=30$ & $\mathrm{~d}_{1 \mathrm{f}}=85.20$ \\
\hline FR14000** & 2 & $D_{1}=300 D_{2}=400$ & $\mathrm{~d}_{1}=10 \quad \mathrm{~d}_{2}=10$ & $\mathrm{~d}_{1 \mathrm{f}}=212.48 \quad \mathrm{~d}_{2 \mathrm{f}}=211.41$ \\
\hline
\end{tabular}

\subsection{Resultados da modelagem M2 - agrupamento de estações}

Analisando as Tabelas 6 e 7, sob o critério de número de variáveis, restrições e número de agrupamentos, também foi possível resolver problemas de dimensões variadas. Além disso, observamos que as restrições de distância foram cumpridas em todos os problemas teste.

Tabela 6 - Informações gerais sobre os problemas teste.

\begin{tabular}{|c|c|c|c|c|c|}
\hline Problema & No de ERB's & No de Pontos & Raio z & Iterações BFGS & $\begin{array}{c}\text { Tempo } \\
\text { CPU(Seg) }\end{array}$ \\
\hline SP80* & 5 & 3153 & 154.2000 & 178 & 92 \\
\hline SP120* & 9 & 6950 & 155.1809 & 140 & 255 \\
\hline SP160* & 12 & 12284 & 99.2575 & 401 & 1672 \\
\hline IT6000** & 9 & 6116 & 72.7549 & 402 & 605 \\
\hline IT7000** & 10 & 7116 & 91.5375 & 442 & 874 \\
\hline IT9000* & 15 & 9115 & 86.9445 & 430 & 2015 \\
\hline
\end{tabular}


Tabela 7 - Número de ERB's por agrupamento, distâncias finais e iniciais.

\begin{tabular}{|c|c|c|c|}
\hline Problema & $\begin{array}{c}\text { № de ERB's } \\
\text { por Agrupamento }\end{array}$ & $\begin{array}{c}\text { Distâncias Máximas } \\
\text { Iniciais } D_{r}\end{array}$ & $\begin{array}{c}\text { Distâncias Máximas } \\
\text { Finais } \bar{D}_{r}\end{array}$ \\
\hline SP80* & $\left|\mathrm{Q}_{1}\right|=3,\left|\mathrm{Q}_{2}\right|=2$ & $D_{1}=100, D_{2}=180$ & $\overline{D_{1}}=99.99, \overline{D_{2}}=92.24$ \\
\hline SP120* & $\left|\mathrm{Q}_{1}\right|=2,\left|\mathrm{Q}_{2}\right|=3,\left|\mathrm{Q}_{3}\right|=4$ & $\begin{array}{c}D_{1}=110, D_{2}=80 \\
D_{3}=50\end{array}$ & $\begin{array}{c}\overline{D_{1}}=109.99, \overline{D_{2}}=74.29 \\
\overline{D_{3}}=47.62\end{array}$ \\
\hline SP160* & $\begin{array}{c}\left|\mathrm{Q}_{1}\right|=2,\left|\mathrm{Q}_{2}\right|=2,\left|\mathrm{Q}_{3}\right|=3, \\
\left|\mathrm{Q}_{4}\right|=5\end{array}$ & $\begin{array}{l}D_{1}=100, D_{2}=200 \\
D_{3}=80, D_{4}=160\end{array}$ & $\begin{array}{l}\overline{D_{1}}=79.78, \overline{D_{2}}=180.32 \\
\overline{D_{3}}=79.99, \overline{D_{4}}=157.96\end{array}$ \\
\hline IT6000** & $\left|\mathrm{Q}_{1}\right|=2,\left|\mathrm{Q}_{2}\right|=3,\left|\mathrm{Q}_{3}\right|=4$ & $\begin{array}{c}D_{1}=120, D_{2}=180 \\
D_{3}=220\end{array}$ & $\begin{array}{c}\overline{D_{1}}=78.32, \overline{D_{2}}=128.99 \\
\overline{D_{3}}=219.64\end{array}$ \\
\hline IT7000** & $\left|\mathrm{Q}_{1}\right|=4,\left|\mathrm{Q}_{2}\right|=6$ & $D_{1}=180, D_{2}=120$ & $\overline{D_{1}}=179.87, \overline{D_{2}}=119.99$ \\
\hline FIT $9000 * *$ & $\begin{array}{c}\left|\mathrm{Q}_{1}\right|=2,\left|\mathrm{Q}_{2}\right|=3,\left|\mathrm{Q}_{3}\right|=4, \\
\left|\mathrm{Q}_{4}\right|=6\end{array}$ & $\begin{array}{l}D_{1}=110, D_{2}=80 \\
D_{1}=40, D_{2}=160\end{array}$ & $\begin{array}{l}\overline{D_{1}}=101.12, \overline{D_{2}}=79.92 \\
\overline{D_{3}}=35.86, \overline{D_{4}}=152.86\end{array}$ \\
\hline
\end{tabular}

*Pontos eqüidistantes **Pontos distribuídos aleatoriamente

\subsection{Resultados da modelagem M3: conjuntos de pontos candidatos para instalação de ERB's}

Analisando as Tabelas 8 e 9, mais uma vez, foi observada a adequação da modelagem para resolver problemas de diferentes dimensões. Além da restrição de cobertura total, destacamos que as restrições de seleção das estações também foram integralmente cumpridas (vide restrições 30.1 e 30.2). Observamos que para todos os problemas teste da Tabela 8 o valor de $\delta$ foi igual a 0.0001 .

Tabela 8 - Informações gerais sobre os problemas teste.

\begin{tabular}{|c|c|c|c|c|c|}
\hline Problema & No de ERB's & No de Pontos & Raio z & Iterações BFGS & $\begin{array}{c}\text { Tempo } \\
\text { CPU(Seg) }\end{array}$ \\
\hline SG80* & 5 & 3697 & 177.9515 & 151 & 177 \\
\hline SG120* & 7 & 8188 & 129.2952 & 79 & 278 \\
\hline SG140* & 3 & 11076 & 214.9794 & 48 & 96 \\
\hline SG4000** & 5 & 4125 & 184.2745 & 142 & 176 \\
\hline SG6000** & 3 & 6125 & 208.2367 & 81 & 89 \\
\hline SG10000** & 8 & 10125 & 129.2962 & 109 & 498 \\
\hline
\end{tabular}

*Pontos eqüidistantes **Pontos distribuídos aleatoriamente 
Tabela 9 - Número de conjuntos $H_{r}$ e de pontos $h_{r l}$ em cada conjunto $H_{r}$.

\begin{tabular}{|c|c|c|}
\hline Problema & No de Conj. $H_{r}$ & $\mathbf{N}^{\mathbf{o}}$ de Pontos $h_{r l}$ em $H_{r}$ \\
\hline SG80* & 5 & $\left|\mathrm{H}_{1}\right|=3,\left|\mathrm{H}_{2}\right|=4,\left|\mathrm{H}_{3}\right|=6,\left|\mathrm{H}_{4}\right|=4,\left|\mathrm{H}_{5}\right|=2$ \\
\hline SG120* & 7 & $\left|\mathrm{H}_{1}\right|=4,\left|\mathrm{H}_{2}\right|=4,\left|\mathrm{H}_{3}\right|=4,\left|\mathrm{H}_{4}\right|=4,\left|\mathrm{H}_{5}\right|=4,\left|\mathrm{H}_{6}\right|=4,\left|\mathrm{H}_{7}\right|=4$ \\
\hline SG140* & 3 & $\left|\mathrm{H}_{1}\right|=3,\left|\mathrm{H}_{2}\right|=3,\left|\mathrm{H}_{3}\right|=3$ \\
\hline SG4000** & 5 & $\left|\mathrm{H}_{1}\right|=5,\left|\mathrm{H}_{2}\right|=5,\left|\mathrm{H}_{3}\right|=5,\left|\mathrm{H}_{4}\right|=5,\left|\mathrm{H}_{5}\right|=5$ \\
\hline SG6000** & 3 & $\left|\mathrm{H}_{1}\right|=2,\left|\mathrm{H}_{2}\right|=4,\left|\mathrm{H}_{3}\right|=6$ \\
\hline SG10000** & 8 & $\left|\mathrm{H}_{1}\right|=2,\left|\mathrm{H}_{2}\right|=2,\left|\mathrm{H}_{3}\right|=2,\left|\mathrm{H}_{4}\right|=2,\left|\mathrm{H}_{5}\right|=2,\left|\mathrm{H}_{6}\right|=2,\left|\mathrm{H}_{7}\right|=2,\left|\mathrm{H}_{8}\right|=2$ \\
\hline \multicolumn{3}{|c|}{ *Pontos eqüidistantes $\quad * *$ Pontos distribuídos aleatoriamente }
\end{tabular}

\section{Conclusões}

A partir do desenvolvimento das modelagens apresentadas na seção 4 e dos resultados apresentados na seção 5, podemos fazer as seguintes considerações:

- A combinação das técnicas de suavização e penalização hiperbólica se mostrou como uma boa alternativa para a resolução da modelagem min-max-min, com uma natureza multi-nível e a característica de não-diferenciabilidade. Com a combinação destas técnicas, podemos transformar o problema min-max-min em um problema diferenciável e, desta forma, podemos utilizar algoritmos que trabalham com as informações das derivadas de segunda ordem.

- Com a utilização desta nova modelagem, tivemos uma significativa redução no número de variáveis associadas ao problema de localização de ERB's. Ou seja, em nossa modelagem temos $2 q+1$ variáveis e nas modelagens ortodoxas temos $q \cdot m+q$ variáveis, sendo $q$ o número de ERB's e $m$ o número de pontos a serem cobertos. Com esta redução, temos a possibilidade de resolver problemas com razoável número de ERB's e pontos a serem cobertos.

- Com o desenvolvimento das três modelagens variantes, podemos considerar outros aspectos relevantes associados ao problema de localização de ERB's. Observamos novamente, em relação ao número de ERB's e ao número de pontos a serem cobertos, que estas modelagens funcionaram perfeitamente para problemas teste de dimensão variada (vide seção 5). Além disso, em todas as modelagens foi garantida a restrição de cobertura total da região $S$.

Em síntese, pelas observações e resultados apresentados, podemos verificar que há uma boa indicação de que a modelagem min-max-min, as suas modelagens variantes e a utilização da teoria de suavização hiperbólica constituem uma boa ferramenta para o problema de localização de ERB's.

\section{Agradecimentos}

Os autores agradecem aos três revisores anônimos pelos valiosos comentários e sugestões e ao professor Flávio Marcelo Tavares Montenegro pela revisão deste trabalho. 


\section{Referências Bibliográficas}

(1) Bazaraa, M.S.; Sherali, H.D \& Shetty, C.M. (1993). NonLinear Programming - Theory and Algorithms. John Wiley \& Sons Publishers, New York.

(2) Beasley, J.E. \& Christofides, N. (1983). Extensions to a Lagrangean Relaxation Approach for the Capacitated Warehouse Location Problem. European Journal of Operational Research, 12(1), 19-28.

(3) Bhaskar, K. \& Wicker, S.B. (2000). Global Search Techniques for Problems in Mobile Communications. In: Telecommunications Optimization: Adaptive and Heuristic Approaches [edited by David Corne et al.], John Wiley \& Sons Publishers.

(4) Bondy, J.A. \& Murty, U.S.R. (1982). Graph Theory with Applications. Elsevier Science Publishing Co., Inc.

(5) Brito, J.A. de M. \& Xavier, A.E. (2004). Algoritmo de Suavização Hiperbólica para o Problema de Localização de Estações de Rádio Base. Anais do XXXVI SOBRAPO. Simpósio Brasileiro de Pesquisa Operacional. São João Del Rei, MG.

(6) Debord, J. (2000). Library of the Mathematical Routines, Laboratorie de Pharmacologie. Faculte de Medicine, Limoges (France). <www.unilim.fr/pages_person/jean.debord/ tpmath//tpmath.htm>.

(7) Dennis, J.E.M. \& Schnabel, R.B. (1994). Numerical Methods for Unconstrained Optimization and NonLinear Equations. SIAM, Classics in Applied Mathematics, 16.

(8) Geoffrion, A.M. \& Graves, G.W. (1974). Multicommodity Distribution System Design by Benders Decomposition. Management Science, 20, 822-844.

(9) Hanif, D.S. (1996). Optimal Location of Transmitters for Micro-Cellular Radio Communications System Design. IEEE, Journal on Selected Areas in Communications, 14, 652-673.

(10) Lages, E.L. (1991). Curso de Análise. Projeto Euclides, IMPA, Livros Técnicos e Científicos.

(11) LeMarechal, C. (1981). Nondifferentiable Optimization. In: Nonlinear Optimization [edited by M.J.D. Powell], Academic Press.

(12) Mateus, G.R.; Cruz, F.R.B. \& Smith, J.M. (1997). Solving to Optimality the Uncapacitated Fixed-Charge Network Flow Problem. Computers and Operations Research, 25(1), 67-81.

(13) Mateus, G.R. (1998). Introdução à Computação Móvel. $11^{\mathrm{a}}$ Escola de Computação.

(14) Mateus, G.R. \& Mazzini, F.F. (2003). Lagrangean Based Methods for Solving Large Scale Cellular Network Design Problems. Wireless Networks, USA, 9(6), 659-672.

(15) Nascimento, J. (2000). Telecomunicações. $2^{\mathrm{a}}$ edição, Makron Books.

(16) Padberg, M.W. (1979). Covering, Packing and Knapsack Problems. Annals of Discrete Mathematics, 4.

(17) Pillo, G.; Grippo, L. \& Lucidi, S. (1993). A Smooth Method for the Finite Minimax Problem. Mathematical Programming, 60, 187-214. 
(18) Polyak, R.A. (1988). Smooth Optimization Methods for the Minimax Problems. SIAM, Journal on Control and Optimization, 26, 1274-1286.

(19) Resende, M.G.C. \& Sousa, J.P. (2004). Metaheuristics: Computer Decision-Making. Kluwer Academic Publishers.

(20) Resende, M.G.C. \& Feo, T.A. (1989). A Probabilistic Heuristic for Computationally Difficult Set Covering Problem. Operations Research Letters, 8, 67-71.

(21) Rosen, J.B. (1983). Global Minimization of a Linearly Constrained Concave Function by Partition of Feasible Domain. Mathematical of the Operational Research, 8(2), 215-230.

(22) Stamatelos, D. \& Epheremides, A. (1996). Spectral Efficiency and Optimal Base Placement for Indoor Wireless Networks. IEEE, Journal on Selected Areas in Communications, 14, 651-661.

(23) Stüber, G.L. (2000). Principles of Mobile Communication. $2^{\mathrm{a}}$ edição, KAP.

(24) Tutschku, K. (1998). Spatial Traffic Estimation and Characterization Mobile Communication Network Design. IEEE, Journal on Selected Areas in Communications, 16.

(25) Wolsey, L.A. \& Fisher, M. (1982). On Greedy Heuristic for Covering and Packing Problems. SIAM, Journal on Algebraic and Discrete Methods, 3, 584-591.

(26) Wolsey, L.A. (1998). Integer Programming. Series in Discrete Mathematics and Optimization. Wiley-Interscience, New York.

(27) Xavier, A.E. \& Oliveira, A. (2005). Optimum Covering of Plane Domains by Circles Via Hyperbolic Smoothing Method. Journal of Global Optimization, 31(3), 493-504.

(28) Xavier, A.E. (2001). Hyperbolic Penality: Method for Nonlinear Programming with Inequalities. International Transactions in Operational Research, 8, 659-672. 